\title{
Generation of Intense Free-Electron Laser Radiation in the Terahertz Regime
}

\author{
Y. Pinhasi*, Y. LuRIE, G.A. Pinhasi AND A. FRIEDman \\ Ariel University, Ariel, Israel
}

(Received May 21, 2015)

\begin{abstract}
Free-electron lasers are high power radiation sources that utilize a distributed interaction between an accelerated electron beam and the electromagnetic field. In these devices, the electron beam serves as the amplification medium generating electromagnetic radiation, while propagating in a periodic magnetic structure called "wiggler" or "undulator". When electrons pass in the wiggler, they oscillate and act as a moving dipole emitting a wave packet of undulator synchrotron radiation. Incoherent summation of the wave packets results in a spontaneous emission. When the electrons are bunched into a short pulse, they all emit their wave packets in the same phase. The radiation wave packets combine coherently, resulting in super-radiance (where the energy radiated is proportional to the square of the electric charge). Such short bunches can be generated by an RF linear accelerator, driven by a photocathode injector. The radiation wavelength is determined by the velocity of the electrons and the spatial period of the undulator. The super-radiance mechanism enables the generation of intense radiation in frequency bands, whereas conventional sources fail to produce a powerful coherent radiation. In this article, we describe the design and analysis of ultrashort pulse free-electron laser operating at the sub-millimeter and terahertz regimes. The free-electron laser is based on a magnetostatic planar wiggler, in which super-radiant emission is emanated by accelerated electron bunches. A three-dimensional, space-frequency theory is developed in order to study radiation excitation in the wiggler. The total electromagnetic field (radiation and space-charge waves) is presented in the frequency domain as an expansion in terms of transverse eigenmodes of the (cold) cavity, in which the field is excited and propagates. The mutual interaction between the electron beam and the electromagnetic field is fully described by coupled equations, expressing the evolution of mode amplitudes and electron beam dynamics. The approach is applied in a numerical particle code WB3D, simulating wide-band interaction of a free-electron laser operating in the linear and non-linear regimes. The model is utilized to study spontaneous and super-radiant emissions radiated by an electron bunch at the sub-millimeter regime, taking into account three-dimensional space-charge effects emerging in such ultrashort bunches.
\end{abstract}

DOI: 10.12693/APhysPolA.128.315

PACS: $41.60 . \mathrm{Cr}, 52.59 . \mathrm{Rz}$

\section{Introduction}

Terahertz radiation is of interest for a basic science, medical and biological applications, spectrometry, remote detection, etc. Among the applications are cancer diagnostics and treatment, medical imaging (especially for dentistry), high bandwidth radars and communications, remote sensing and imaging, fast spectroscopy, and radiative power beaming. Utilization of $\mathrm{THz}$ radiation is becoming wider as instantaneous power terahertz sources, like free-electron lasers (FELs) are developed.

FELs are electron devices utilizing distributed interaction between an electron beam and electromagnetic radiation. Such devices provide intense coherent electromagnetic radiation over a wide range of $\mathrm{THz}$ frequencies. When the electrons enter the wiggler, they oscillate and emit undulator synchrotron radiation. Random electron distribution in the e-beam cause fluctuations in current density, identified as shot noise in the beam current. The electromagnetic fields excited by each electron add incoherently, resulting in a spontaneous emission.

\footnotetext{
${ }^{*}$ corresponding author; e-mail: yosip@ariel.ac.il
}

In high-gain FELs, utilizing sufficiently long undulators, the spontaneous emission radiation excited in the first part of the undulator is amplified along the reminder of the interaction region resulting in synchrotronamplified spontaneous emission (SASE). Such well-known FEL mechanism is widely used for generation of far ultraviolet and X-ray wavelengths but also for the production of infrared and THz radiation. In SASE FELs, an initial spontaneous emission electron beam is self amplified along the wiggler, giving rise to non-linear beamradiation interaction. This results in an exponential growth of the emission up to saturation.

When the electron beam is modulated or pre-bunched, the fields excited by the electrons become correlated, and coherent summation of radiation fields from individual particles occurs. If all electrons radiate in phase with each other, the generated radiation becomes coherent super-radiant emission [1], resulting in an enhancement of undulator super-radiant emission of short electron pulses. Energy chirping is a well-known technique used in accelerator physics for density compression of electron pulses. In an ESR FEL, it is suggested to cause a longitudinal density compression of short electron pulses just inside the FEL interaction region, i.e. inside the wiggler. 


\section{TABLE}

Operational parameters for the Israeli THz FEL.

\begin{tabular}{c|c}
\hline \hline \multicolumn{2}{c}{ Accelerator } \\
\hline type & photo-injector RF-LINAC \\
beam energy & $E_{\mathrm{k}}=5.5 \mathrm{MeV}$ \\
pulse duration & $T_{\mathrm{b}}>0.1 \mathrm{ps}$ \\
bunch charge & $Q_{\mathrm{b}}=30-500 \mathrm{pC}$ \\
\hline \multicolumn{2}{|c}{ Wiggler } \\
\hline magnetic induction & $B_{\mathrm{w}}=2 \mathrm{kG}$ \\
period & $\lambda_{\mathrm{w}}=25 \mathrm{~mm}$ \\
\hline \multicolumn{2}{|c}{ Waveguide } \\
\hline rectangular & $15 \times 10 \mathrm{~mm}^{2}$
\end{tabular}

In the present work, we consider an FEL which is designed to be driven by trains of short electron pulses, produced by a photo-injector RF linear accelerator (RFLINAC). The Israeli RF-LINAC FEL project under development is considered here as an example of such a radiation source [2]. Basic operational parameters of the FEL are given in Table. Two possible configurations of the FEL are considered and compared in the work: a self amplified spontaneous emission (SASE) FEL, and an enhanced super-radiance (ESR) FEL [3].

\section{The simulation model}

To simulate and compare between both FEL configurations, 3D space-frequency approach [4] was applied. The method is based on an expansion of the highfrequency electromagnetic field in terms of transverse eigenmodes of the medium (free-space or a waveguide) in which the field is excited and propagates. The perpendicular component of the electric and magnetic fields are given in any cross-section as a linear superposition of a complete set of transverse eigenmodes

$$
\begin{aligned}
& \tilde{\boldsymbol{E}}_{\perp}(\boldsymbol{r}, f)= \\
& \sum_{q}\left[C_{+q}(z, f) \mathrm{e}^{-\mathrm{i} k_{z q} z}+C_{-q}(z, f) \mathrm{e}^{+\mathrm{i} k_{z q} z}\right] \tilde{\mathcal{E}}_{q \perp}(x, y), \\
& \tilde{\boldsymbol{H}}_{\perp}(\boldsymbol{r}, f)= \\
& \sum_{q}\left[C_{+q}(z, f) \mathrm{e}^{-\mathrm{i} k_{z q} z}-C_{-q}(z, f) \mathrm{e}^{+\mathrm{i} k_{z q} z}\right] \tilde{\mathcal{H}}_{q \perp}(x, y) .
\end{aligned}
$$

$C_{+q}(z, f)$ and $C_{-q}(z, f)$ are scalar amplitudes of the $q$-th forward and backward modes, respectively, with electric field $\tilde{\mathcal{E}}_{q \perp}(x, y)$ and magnetic field $\tilde{\mathcal{H}}_{q \perp}(x, y)$ profiles and axial wave number

$k_{z q}=\left\{\begin{array}{l}\sqrt{k^{2}-k_{\perp q}^{2}}, \quad k>k_{\perp q} \text { (propagating modes) }, \\ \mathrm{i} \sqrt{k_{\perp q}^{2}-k^{2}}, \quad k<k_{\perp q} \text { (cut-off modes). }\end{array}\right.$

Expressions for the longitudinal component of the electric and magnetic fields are obtained after substituting the modal representation of the perpendicular fields into Maxwell's equations, where source of electric current density $\tilde{\boldsymbol{J}}(\boldsymbol{r}, f)$ is introduced

$$
\tilde{E}_{z}(\boldsymbol{r}, f)=\sum_{q}\left[C_{+q}(z, f) \mathrm{e}^{-\mathrm{i} k_{z q} z}\right.
$$

$$
\begin{aligned}
& \left.-C_{-q}(z, f) \mathrm{e}^{+\mathrm{i} k_{z q} z}\right] \tilde{\mathcal{E}}_{q z}(x, y)+\frac{\mathrm{i}}{2 \pi f \varepsilon_{0}} \tilde{J}_{z}(\boldsymbol{r}, f), \\
& \tilde{H}_{z}(\boldsymbol{r}, f)=\sum_{q}\left[C_{+q}(z, f) \mathrm{e}^{-\mathrm{i} k_{z q} z}\right. \\
& \left.\quad+C_{-q}(z, f) \mathrm{e}^{+\mathrm{i} k_{z q} z}\right] \tilde{\mathcal{H}}_{q z}(x, y) .
\end{aligned}
$$

The interaction between the electromagnetic field and the gain medium is fully described by a set of coupled equations, expressing the evolution of mode amplitudes along the interaction region

$$
\begin{gathered}
\frac{\mathrm{d}}{\mathrm{d} z} C_{ \pm q}(z, f)= \\
\mp \frac{\mathrm{e}^{ \pm \mathrm{i} k_{z q} z}}{\mathcal{N}_{q}} \iint\left[\frac{Z_{q}}{Z_{q}^{*}} \tilde{\boldsymbol{J}}_{\perp}(\boldsymbol{r}, f) \pm \hat{\boldsymbol{z}} \tilde{J}_{z}(\boldsymbol{r}, f)\right] \tilde{\mathcal{E}}_{q}^{*}(x, y) \mathrm{d} x \mathrm{~d} y .
\end{gathered}
$$

The normalization of the field amplitudes of each mode is made via each mode's complex Poynting vector power

$$
N_{q}=\iint\left[\tilde{\mathcal{E}}_{q_{\perp}}(x, y) \times \tilde{\mathcal{H}}_{q_{\perp}}^{*}(x, y)\right] \hat{\boldsymbol{z}} \mathrm{d} x \mathrm{~d} y
$$

and the mode impedance is given by

$$
Z_{q}=\left\{\begin{array}{l}
\sqrt{\frac{\mu}{\varepsilon}} \frac{k}{k_{z q}}=\frac{2 \pi f \mu_{0}}{k_{z q}} \text { for TE modes, } \\
\sqrt{\frac{\mu}{\varepsilon}} \frac{k_{z q}}{k}=\frac{k_{z q}}{2 \pi f \varepsilon_{0}} \text { for TM modes. }
\end{array}\right.
$$

The model was realized in a numerical code WB3D and has been successfully applied to the analysis of various effects in FEL devices [5-11]. The electron beam pulse is considered in the model as a consisting of charged electron "macro-clusters" distributed over the beam. An initial distribution of the clusters in the beam is of great importance in simulations of radiation emission processes. Random Gauss longitudinal distribution of a fixed number $\left(N_{q}=300\right)$ of equal charges was considered in the present work. Unfortunately this approach results in an artificial level of the beam spontaneous emission due to introduction of "electron clusters" instead of real electrons, which prevents from a correct description of radiation build-up process starting from spontaneous emission of a noisy drive electron beam. However this simple model seems to be adequate enough in description of a coherent emission of ultrashort (shorter than the radiation wavelength) or bunched electron beam. To improve the description of spontaneous emission and of transition effects, unified model of electron beam short noise from [12] was also applied to simulate the initial electron beam short noise in a wide frequency range.

\section{Numerical results}

The simulations were carried out supposing the beam energy of $E_{\mathrm{k}}=5.5 \mathrm{MeV}$, which corresponds to the FEL synchronism frequency of $f_{s} \approx 2.96 \mathrm{THz}\left(\lambda_{s} \approx 100 \mu \mathrm{m}\right)$. Typical spectrum of SASE emission is illustrated in Fig. 1 where an example of SASE radiation spectrum obtained at different points of the wiggler is shown. The example corresponds to the $Q_{\mathrm{b}}=0.5 \mathrm{nC}$ electron beam pulse of relative duration $f_{s} T_{\mathrm{b}} \approx 2$. A unified model of electron 
beam short noise was applied in order to simulate an initial distribution of 3501 clusters. The total power of the emitted radiation is demonstrated in Fig. 2 as function of the wiggler length (in the units of the wiggler's period). The results presented were received in the unified model of electron beam short noise for electron beam pulses of the charges of $Q_{\mathrm{b}}=0.1,0.5$, and $1.0 \mathrm{nC}$. Convergence of the results with the number of macro-clusters considered in the simulations is also shown.

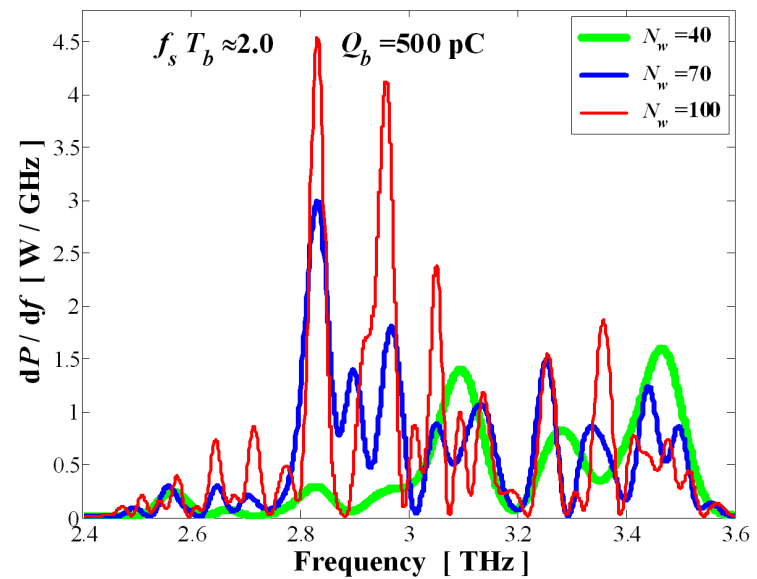

Fig. 1. Example of SASE radiation spectrum obtained at different points of the wiggler.

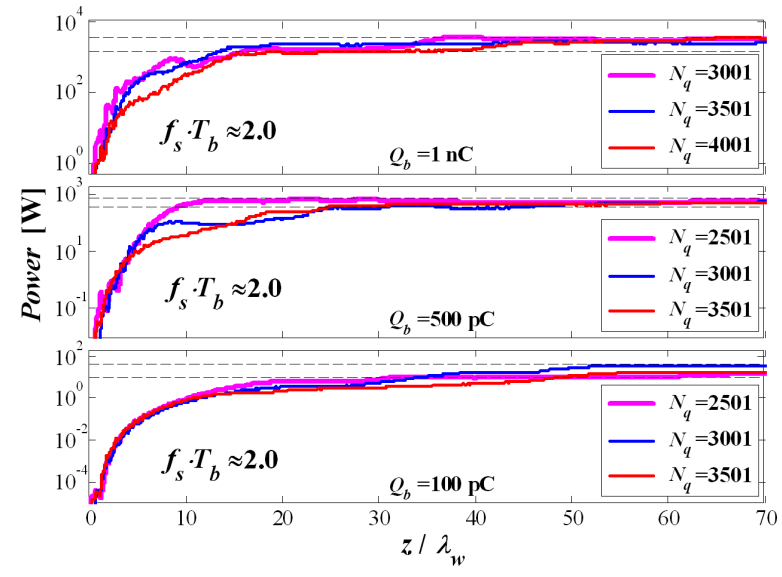

Fig. 2. SASE FEL power build-up.

Enhanced super-radiance can be achieved in FELs driven by a train of short electron pulses which are subjected to a controlled energy chirp. Coherent emission of an electron bunch is strongly depressed if the bunch is about the radiation wavelength long or longer. Energy chirp gives rise to a longitudinal density compression of the bunch just while it propagates along the wiggler, enhancing super-radiance [3]. We consider such FEL configuration as a competitor to SASE in construction of compact FELs for the terahertz frequency spectral range. Simulations of ESR FEL were carried out supposing the $L_{\mathrm{w}}=1 \mathrm{~m}$ long wiggler $\left(N_{\mathrm{w}}=40\right.$ periods); a shorter wiggler can actually be taken, depending on

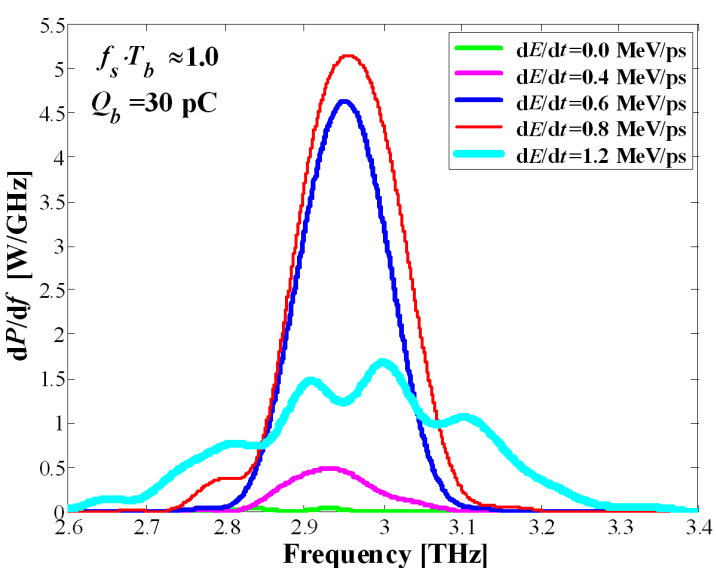

Fig. 3. Radiation spectrum of ESR FEL.

the initial beam pulse duration, and on the energy chirp rate. Figure 3 demonstrates the radiation spectrum of ESR FEL, obtained in the example of the beam charge of $Q_{\mathrm{b}}=30 \mathrm{pC}$, and of the initial relative beam pulse duration $f_{s} T_{\mathrm{b}} \approx 1$. A proper choice of the energy chirp rate $(\mathrm{d} E / \mathrm{d} t \geq 0.6 \mathrm{MeV} / \mathrm{ps}$ in the example given) provides a remarkable increase in the total radiation power (about two orders of magnitude) as one can see in the picture. This energy chirp rate level means the total energy variance during the beam pulse of

$$
\Delta E / E_{\mathrm{b}}=\frac{1}{E_{\mathrm{b}}} \frac{\mathrm{d} E}{\mathrm{~d} t} T_{\mathrm{b}} \approx 3.6 \% .
$$

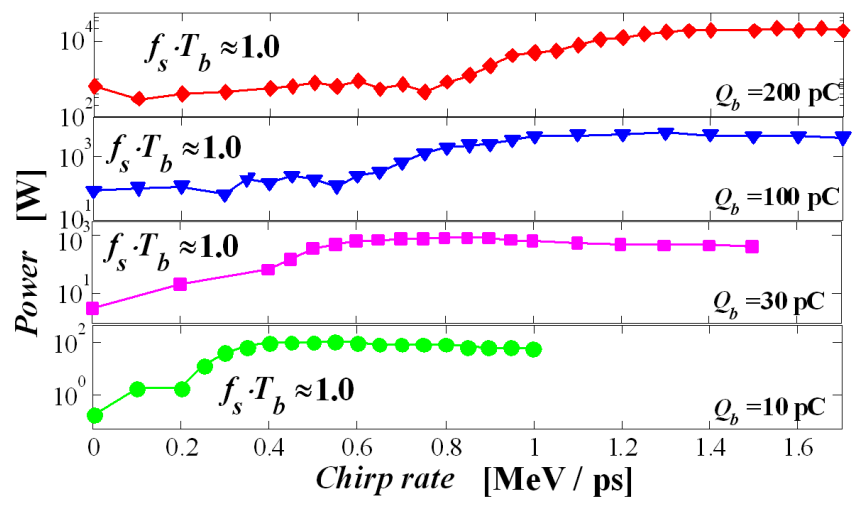

Fig. 4. ESR FEL power dependence on the chirp rate.

Such energy chirping can be achieved in RF-LINAC FEL scheme by means of some phase de-synchronization of the accelerated electron pulses relative to the accelerating electromagnetic wave in the RF-LINAC cavities, similar to the application of the "phase-matching" section within the FEL beam line [1]. Figure 4 shows dependence of the radiation power on the energy chirp rate used.

Radiation power obtained in the both FEL configurations considered is presented in Fig. 5 as a function of the beam charge. Up to considerably high beam charges where space-charge effects reduce a coherent emission, 


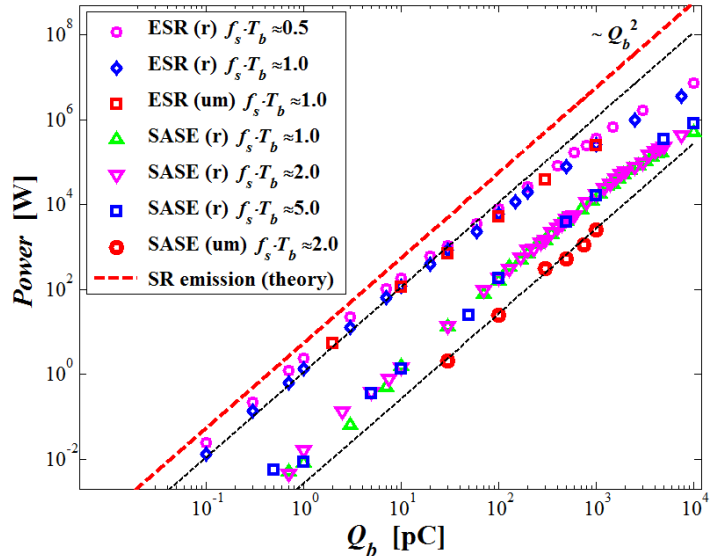

Fig. 5. Radiation power of ESR and SASE FELs as a function of the beam charge.

the power in both FEL configurations seems to grow linearly with the beam charge squared which is characteristic to super-radiant emission process.

\section{Conclusions}

Simulations of SASE FEL with initial random Gauss distribution of macro-charges seem to overestimate the saturation power and the saturation length. The unified model of electron beam short noise [12] is applied instead to a more realistic description of an initial spontaneous emission of noisy drive beam (short noise) and of the transition radiation build-up process. In contrast, nearly the same results were received in simulations of ESR FEL in both models. This difference can be explained by a much stronger bunching taking place in ESR FEL (bunching factor of about 5-6\% in comparison with more than $90 \%$ at SASE and at ESR FELs, respectively). In ESR FEL, most of the radiation is emitted coherently at the moment of a maximum density compression of the beam pulse; spontaneous emission has a negligible effect at this moment.

A considerably long (about 100 periods) wiggler is required for a SASE FEL in order to enable saturation. The beam bunching is found to be rather weak, and the power of the emitted radiation is much lower than that of ESR FEL driven by electron bunches with similar charges. Spectrum of SASE emission includes a number of narrow peaks, competing each other at saturation. At the range of the beam charges considered, the power of saturated SASE emission is found to demonstrate a super-radiant proportionality to the beam charge squared.

An ESR FEL utilizes short (the radiation wavelength or shorter) electron bunches with a controlled energy chirping. Train of such bunches can be obtained by means of a photo-injector RF-LINAC enabling some phase desynchronization of the accelerated electron pulses relative to the accelerating wave. At a proper chirping, ESR FEL is found to provide a high beam bunching, promising a high efficiency of such radiation source. Unfortunately, destructive space-charge forces emerge to reduce ESR FEL efficiency if overcharged bunches are applied. To overcome this limitation, lowcharged bunches at a higher bunch repetition rate can be used.

The coherence of the resulted ESR is dependent on the effective wiggler length and chirp rate. Spectral quality of the ESR FEL radiation is similar to that of other single-pass FEL configurations. Utilization of a resonating structure improves coherent features of excited radiation. Concluding, ESR FEL seems to be a perspective concept for construction of a photo-injector, $\mathrm{THz}$ spectral range RF-LINAC FEL being under development in Ariel university in cooperation with UCLA.

\section{References}

[1] A. Doria, G.P. Gallerano, E. Giovenale, S. Letardi, G. Messina, C. Ronsivalle, Phys. Rev. Lett. 80 , 2841 (1998); Nucl. Instrum. Methods Phys. Res. A 475, 296 (2001); Phys. Rev. Lett. 93, 264801 (2004).

[2] E. Dyunin, Yu. Lurie, Y. Pinhasi, A. Gover, H. Marks, in: IEEE 25th Convention of Electrical and Electronics Engineers in Israel, Eilat, 2008, p. 825; A. Friedman, N. Balal, V. Bratman, E. Dyunin, Y. Lurie, E. Magory, A. Gover, in: Proc. 36th Int. Free Electron Laser Conf., Basel (Switzerland), 2014, p. 553.

[3] Yu. Lurie, Y. Pinhasi, Phys. Rev. ST Accel. Beams 10, 080703 (2007).

[4] Y. Pinhasi, Yu. Lurie, A. Yahalom, Phys. Rev. E 71, 036503 (2005).

[5] Y. Pinhasi, Yu. Lurie, A. Yahalom, Nucl. Instrum. Methods Phys. Res. A 475, 147 (2001).

[6] Y. Pinhasi, Yu. Lurie, Phys. Rev. E 65, 026501 (2002).

[7] Y. Pinhasi, Yu. Lurie, A. Yahalom, A. Abramovich, Nucl. Instrum. Methods Phys. Res. A 483, 510 (2002).

[8] Y. Pinhasi, Y. Lurie, A. Yahalom, Nucl. Instrum. Methods Phys. Res. A 528, 62 (2004).

[9] A. Gover, E. Dyunin, Y. Lurie, Y. Pinhasi, M.V. Krongauz, Phys. Rev. ST Accel. Beams 8, 030702 (2005).

[10] Yu. Lurie, Y. Pinhasi, Phys. Rev. ST Accel. Beams 13, 050701 (2010).

[11] V. Zhaunerchyk, R.T. Jongma, Yu. Lurie, Y. Pinhasi, W.J. van der Zande, Appl. Phys. Lett. 97, 231109 (2010).

[12] B.W.J. McNeil, M.W. Poole, G.R.M. Robb, Phys. Rev. ST Accel. Beams 6, 070701 (2003). 